J. Astrophys. Astr. (), 000-000

\title{
Radio relics in cosmological simulations
}

M. Hoeft*

Thüringer Landessternwarte, Sternwarte 5, 07778, Tautenburg, Germany

S. E. Nuza \& S. Gottlöber

Leibniz-Institut für Astrophysik Potsdam, An der Sternwarte 16, 14482 Potsdam, Germany

R. J. van Weeren \& H. J. A. Röttgering

Sterrewacht Leiden, PO Box 9513, 2300 RA Leiden, The Netherlands

M. Brüggen

Jacobs University Bremen, Campus Ring 1, 28725 Bremen, Germany

Received xxx; accepted xxx

\begin{abstract}
.
Radio relics have been discovered in many galaxy clusters. They are believed to trace shock fronts induced by cluster mergers. Cosmological simulations allow to study merger shocks in detail since the intra-cluster medium is heated by shock dissipation. Using high resolution cosmological simulations, identifying shock fronts and applying a parametric model for the radio emission allows us to simulate the formation of radio relics. We analyze a simulated shock front in detail. We find a rather broad Mach number distribution. The Mach number affects strongly the number density of relativistic electrons in the downstream area, hence, the radio luminosity varies significantly across the shock surface. The abundance of radio relics can be modeled with the help of the radio power probability distribution which aims at predicting radio relic number counts. Since the actual electron acceleration efficiency is not known, predictions for the number counts need to be normalized by the observed number of radio relics. For the characteristics of upcoming low frequency surveys we find that about thousand relics are awaiting discovery.
\end{abstract}

Key words: Cosmology: large-scale structure of Universe Galaxies: Clusters: general, intracluster medium

*e-mail: hoeft@tls-tautenburg.de 


\section{Introduction}

In many galaxy clusters diffuse radio emission has been discovered in the periphery of the cluster. If the emission has no optical counterpart, i.e. it is apparently not related to an active galaxy, the diffuse emission is classified as a 'radio relic'. Initially it was assumed that they corresponded to the left-overs from former AGN activity, justifying the name 'relic'. Ensslin et al. (1998) instead suggested that they trace merger shock fronts in the intracluster medium (ICM). Within the past years several spectacular relics have been discovered, e.g. the very bright relic in Abell 3667 (Röttgering et al. 1997), the almost ring-like structure in Abell 3376 (Bagchi et al. 2006), the about $2 \mathrm{Mpc}$ long but exceptionally narrow relic in CIZA 2242 (van Weeren et al. 2010), and recently a double relic in a cluster which has been discovered by its Sunyaev-Zeldovich signature, PLCK G287.0+32.9 (Bagchi et al. 2011).

All mentioned radio relics have morphologies similar to shock fronts in cluster simulations (e.g. Paul et al. 2011). Typically, relics are aligned with X-ray isophotes. For a few clusters a shock front in the ICM has been identified in X-ray observations (Markevitch 2010). For instance, for A 3667 the front coincides with the outer edge of the north-west relic. Moreover, the temperature jump has been measured. Rankine-Hugoniot jump conditions for hydrodynamical shock fronts allow us to estimate the Mach number of the shock. Since the ICM temperature is correlated with the mass of the cluster, which also determines the merger velocity, Mach numbers of merger shocks are in the range of 2 to 4 , at maximum.

Cosmological simulations are ideal to study the formation of merger shocks. Since the clusters are heated by the shock fronts any hydrodynamical simulation needs to include shock dissipation. Ryu et al. (2003) introduced the distinction between 'accretion shocks', where photo-ionized gas is shockheated for the first time and 'merger shocks', where the upstream gas has already encountered earlier phases of shock-heating. This clearly limits the Mach numbers of merger shocks. Even if cosmological hydrodynamical codes cover properly the shock dissipation, they do usually not determine the Mach number explicitly. For Smoothed-Particle Hydrodynamics (SPH) Pfrommer et al. (2006) developed a method based on the entropy gain of the particles. Also for SPH, we developed a method based on the entropy gradients (Hoeft et al. 2008). Skillman et al. (2010) used a shock detection method for Eulerian simulations to study radio relics. Recently, Vazza et al. (2009) developed a method to increase the level of refinement in adaptive-mesh refinement simulations at the locations of shock fronts. Hence, even if merger shocks are basically covered in any hydrodynamical cosmological simulation, locating the shocks, determining the Mach number, and achieving sufficient numerical resolution is still a challenge.

A clear relic example has been found in CIZA 2242 (van Weeren et al. 
2010). First, it has an overall spectrum which is close to a power-law and has a slope of $-1.08 \pm 0.05$. Secondly, the clear spectral gradient across the relic supports that electrons get accelerated at the front and then lose energy while moving away from the front. Finally, the spectral aging of the electrons is imprinted as spectral steeping in the radio emission. This summarizes what is currently widely believed as the formation scenario for relics: At the shock fronts electrons get accelerated via diffusive shock acceleration (DSA, Drury 1983; Blandford \& Eichler, 1987; Malkov \& Drury, 2001), with a maximal slope in the radio spectrum of -0.5. The relativistic electrons advect downstream and cool, hence the spectrum steepens with increasing distance from the shock front. However, alternative scenarios has been suggested, see e.g. Keshet (2011).

In this paper we present current efforts to model radio relics in cosmological simulations. First, we summarize how to model radio relics. Secondly, we present results from a recent high resolution simulation. Finally, we briefly introduce predictions for upcoming low-frequency radio surveys.

\section{Modeling the radio emission of relics}

Apparently, radio relics in galaxy clusters are closely related to merger shock fronts. In a plausible scenario for the formation of the radio relics a small fraction of thermal electrons gets accelerated at the front and the resulting relativistic electrons emit synchrotron radiation while advecting downstream. Based on this scenario a radio relic model to be implemented in cosmological simulations can be decomposed into two parts, namely electron acceleration at the shock front and downstream synchrotron emission.

Given the plasma conditions of the ICM in the periphery of galaxy clusters, namely electron densities $\sim 10^{-4} \mathrm{~cm}^{-3}$, temperatures of a few $\mathrm{keV}$, and magnetic fields of the order of $1 \mu \mathrm{G}$, the shock fronts are collisionless, i.e. the energy dissipation cannot be mediated via particle collisions. For rather low Mach numbers anomalous resistivity may be sufficient to dissipate the kinetic energy of the upstream flow. When the Mach number is above the critical Mach number of 2.76 proton reflection at the shock front may cause the necessary dissipation. The detailed processes at the shock front are complex, see e.g. Treumann (2009) for a review of collisonless shocks. The characteristic length scale for the shock transition region is the proton gyroradius. For thermal protons in the periphery of galaxy clusters this radius is of the order of $1 \mathrm{~cm}$, i.e. even less than the mean particle separation. Electrons may get accelerated first via electric fields inherent to the shock structure and higher energies may be achieved via diffusive shock acceleration. In any case, radio relics -observationally and in simulations- represent 


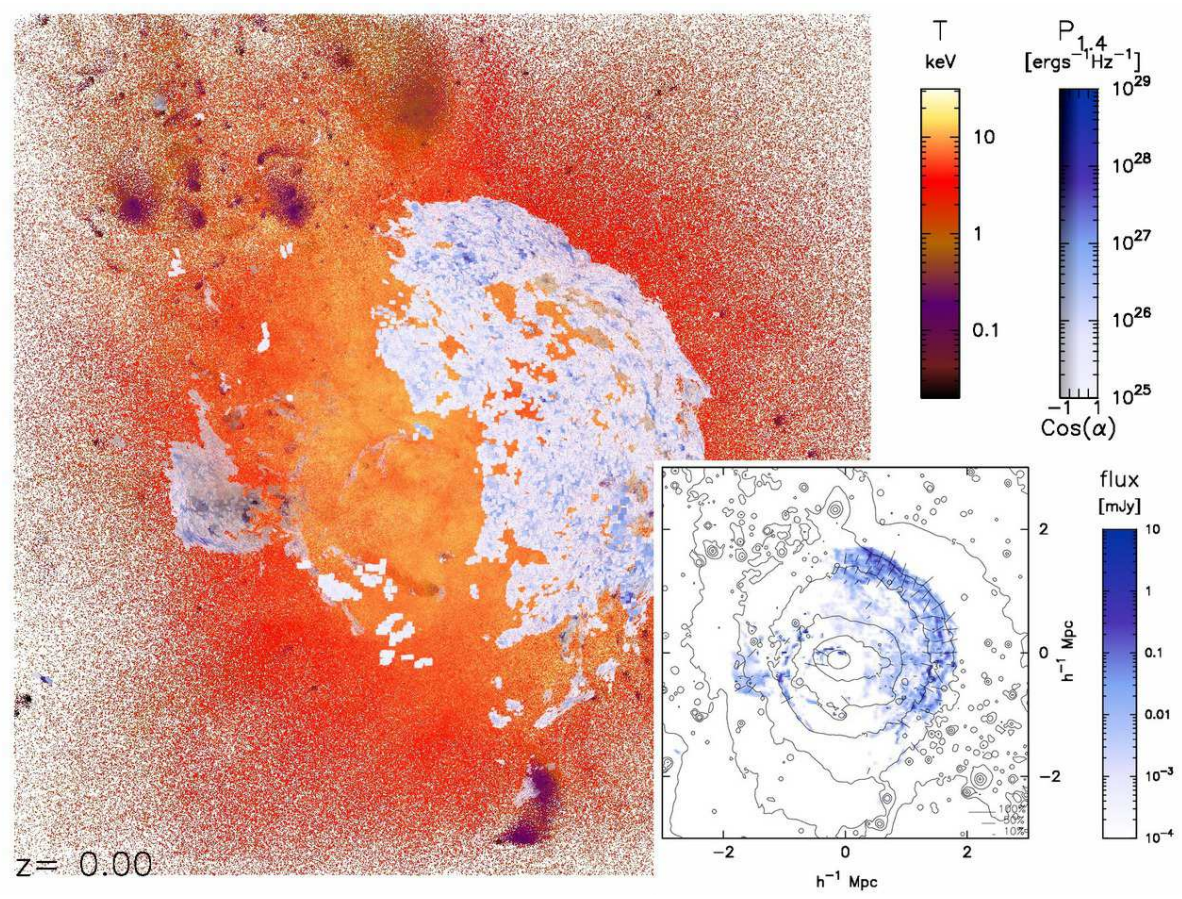

Figure 1. Visualization of the shock front: Particles in the simulation which have been identified as being part of a shock depicted with small white/blue squares. Other particles and those for which the Mach number is very low are depicted as brown/red/yellow dots. The shock fronts has roughly a shape of a shell segment. However, there are many small scale variations in shape and radio luminosity. The lower right inset shows the projected X-ray (contours) and radio emission.

an averaging over a huge shock area, compared to the characteristic shock transition scale, namely the proton gyroradius.

Including the detailed physics of the electron acceleration at the shock front is beyond the scope of any current simulation of galaxy cluster mergers. Instead, the resulting distribution of relativistic electrons has to be implemented as a parametric subgrid model. In Hoeft \& Brüggen (2007) we constructed the following model: The slope of the average electron population is given by the predictions of DSA in the test-particle regime (Drury 1983; Blandford \& Eichler, 1987; Malkov \& Drury, 2001). We assume that this reflects the electron spectrum averaged over a resolution element in the simulation. Moreover, we assumed that a fraction $\xi_{\mathrm{e}}$ of the energy dissipated at the shock front is transferred to the supra-thermal electrons. This allows us to normalize the electron spectrum. However, a lower energy cut-off, $E_{\text {low }}$, is needed for that. We argued that the energy fraction $\xi_{\mathrm{e}}$ refers to any energy above the thermal pool, hence $E_{\text {low }}$ is the energy where the power law distribution meets the thermal one. Based on this condition $E_{\text {low }}$ can be 
determined for given gas temperature, $\xi_{\mathrm{e}}$ and slope of the electron distribution. In our model we have also defined a function $\Psi(\mathcal{M})$ which reflects the dependence of the radio emission as a function of the Mach number. We find that the Mach number needs to be above 2-3 to cause a significant amount of radio emission.

The initial electron spectrum 'ages' while the plasma moves away from the shock front. Electrons in the energy range relevant for the radio emission cool due to synchrotron emission and inverse Compton (IC) losses. Hence the evolution of the spectrum can be modeled at least numerically. However, we can only speculate about the strength and the structure of magnetic fields in the periphery of galaxy clusters. The upper limit for IC emission in the northwest relic in A 3667 puts a lower limit on the magnetic field in that region, namely $\gtrsim 2 \mu \mathrm{G}$ (Nakazawa et al. 2009). The narrowness of the relic in CIZA 2242 indicates that the magnetic field should be $\gtrsim 5 \mu \mathrm{G}$, if we exclude the $B<1 \mu \mathrm{G}$ solution (van Weeren et al. 2010). Rotation Measure analysis of the magnetic field in the Coma cluster indicates that the magnetic field scales with the electron density as $B=4.7 \mu \mathrm{G}\left(n_{\mathrm{e}} / 3.44 \times 10^{-3} \mathrm{~cm}^{-3}\right)^{0.5}$ (Bonafede et al. 2010).

The width of the relic in CIZA 2242 shows that the cooling of the electron population takes place at maximum on scales of the order of $10 \mathrm{kpc}$. Hence to resolve the cooling in the simulation in the relic region a numerical resolution significantly below $10 \mathrm{kpc}$ is needed. For a typical shock speed of $2000 \mathrm{~km} \mathrm{~s}^{-1}$ this implies a time resolution of $\sim 5 \mathrm{Myr}$. Moreover, cooling of the relativistic electrons takes place on even shorter time scales. Since the resolution in our simulations is $\gtrsim 10 \mathrm{kpc}$ we treat the radio emission entirely as a subgrid model. We compute the radio power per shock surface given the Mach number and the downstream properties.

\section{Emission maps in a cosmological simulation}

We aim to study the formation of radio relics in realistic cosmological scenarios. We simulate the cluster formation using the TreeSPH code GADGET (Springel 2005), including hydrodynamics but no radiative cooling or heating processes. To obtain sufficiently massive clusters we use a simulation box with a side length of $160 h^{-1} \mathrm{Mpc}$ and with an initial resolution of $256^{3}$ particles. To increase the effective resolution we apply the zoom-in technique achieving an effective resolution of $2048^{3}$. At the location of radio relics the overdensity is typically of the order of $10^{3}$. With 64 particles in the SPH kernel the resulting hydrodynamical resolution is about $30 \mathrm{~h}^{-1} \mathrm{kpc}$. Therefore, as stated above, treating the downstream aging of electrons accelerated at the shock front entirely as a subgrid model is appropriate.

The ICM medium is heated by the energy dissipation of accretion and merger shock fronts. Hence, any simulation of the ICM needs to account for 

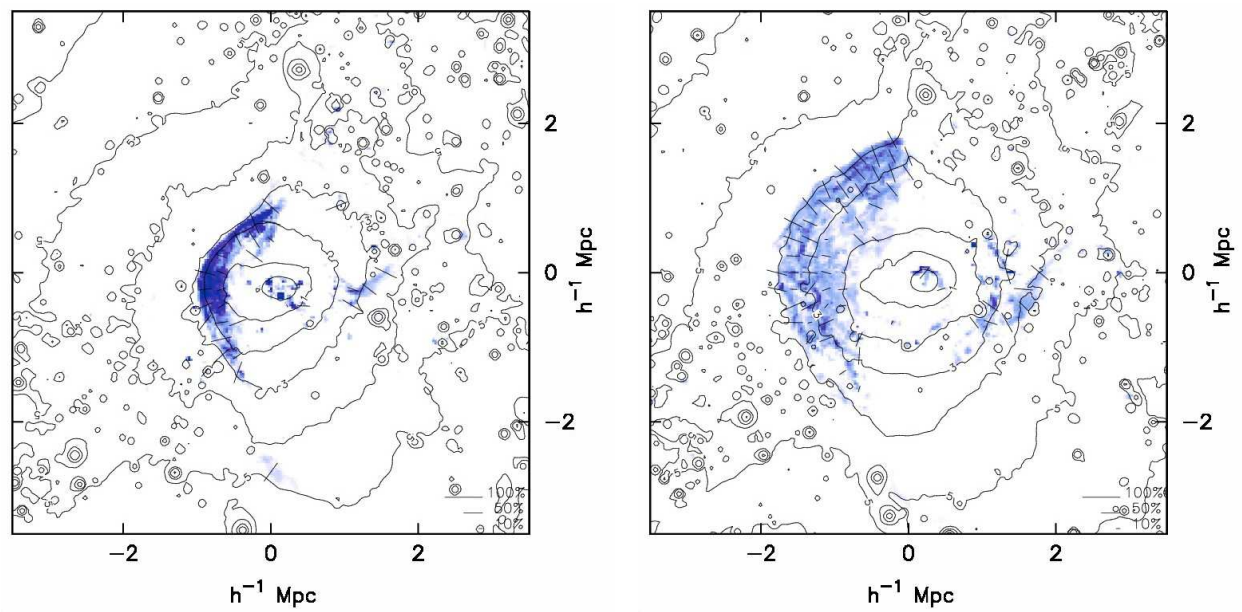

Figure 2. Evolution of the radio relic caused by a merger shock front. One can clearly see how the surface brightness of the relics fades from the early state (left panel) to the later state (right panel, about $300 \mathrm{Myr}$ later), even if the Mach number actually increases.

shock dissipation, but in SPH the Mach number is not explicitly determined. For our radio emission subgrid model we need both, the Mach number and the surface area of the shock front. In Hoeft et al. (2008) we have presented a scheme for determining the Mach number in SPH simulations. In a slightly revised version of the scheme we determine candidate shock fronts using the pressure gradient. A conservative estimate for the Mach number is obtained from the velocity divergence and density and entropy jumps (see Nuza et al. 2011).

To display the shock surface we use the following method. Since we determine the shock normal we can assign to each particle with a radio emission above a minimum value a small square with the shock normal perpendicular on it. The side length of the square is proportional to the $\mathrm{SPH}$ smoothing length of the particle. The combination of all the small squares represents the shock surface, as can be seen in Fig. 1. With a 3D rendering the geometry of the shocks can be easily recognized. The simulations allows also to study how radio relics evolve. We find that our radio relic is very bright right after the first core passage of the cluster merger (left panel) and then continuously decreases in brightness (right panel), see Fig. 2. ${ }^{1}$

We determine the Mach number distribution $\mathrm{d} S / \mathrm{d} \log \mathcal{M}$ in the region of the merger shock, see Fig. 3 (solid line). The distributions peaks at the Mach number of the shock, 2.5, as obtained from analyzing the jump in the X-ray

\footnotetext{
${ }^{1}$ Animations can be found on www.tls-tautenburg.de/research/hoeft/research_struc_shock.html
} 


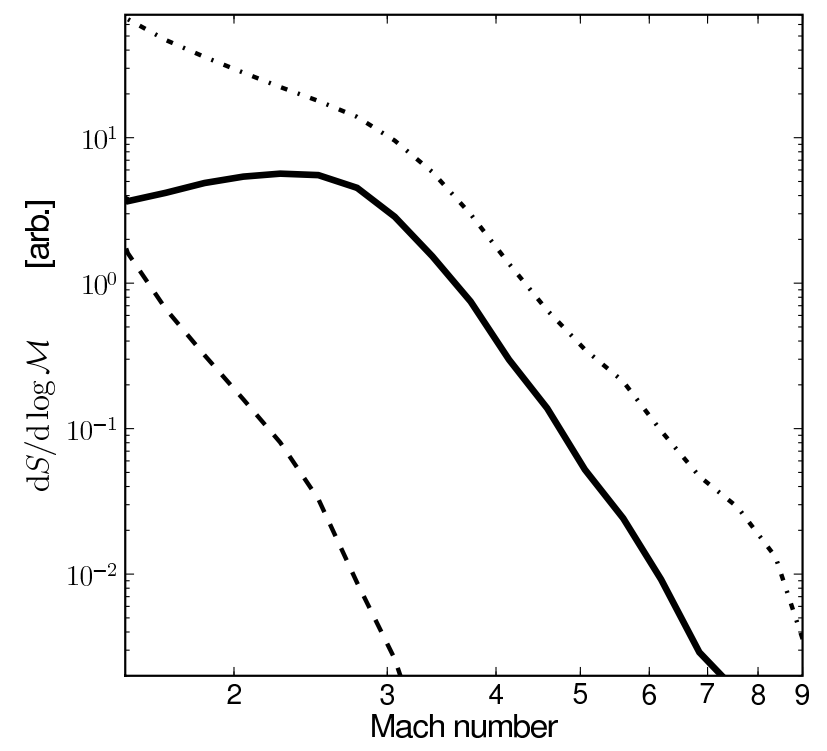

Figure 3. Distribution of Mach numbers $\mathrm{d} S / \mathrm{d} \log \mathcal{M}$, where $S$ is the shock surface area. We have selected a section of a spherical shell which includes the merger shock front as shown in Fig. 1 and have determined with our shock finder the Mach number distribution (solid line). From our modeling of X-ray observations we expect a Mach number of 2.5 from the temperature jump in the projected emission map. For comparison we show the Mach number distribution in the entire cluster up to a radius of $1 \mathrm{Mpc}$ (dashed line) and up to $3 \mathrm{Mpc}$ (dash-dotted line).

emission. However, the distribution is rather broad and becomes almost a power law for $\mathcal{M} \gtrsim 4$. The Mach number varies due to upstream differences in density and temperature. As a consequence the radio luminosity varies significantly across the shock surface.

\section{How many relics can be found?}

Currently, there are about three dozen galaxy clusters known which show one or more radio relic. This allows to start analyzing relics in a statistical way. A natural approach would be to determine a 'radio luminosity function of shock fronts'. More precisely, cosmological simulations should provide a list of shocks which then can be populated with relics, similar to populating dark matter halos with galaxies. However, as shown above, shock fronts have complex geometries and display a broad Mach number distribution. So we cannot simply sort shock fronts, e.g. according to their Mach number, and then distribute the luminous relics to the shock fronts with the highest 
Mach numbers. In Nuza et al. (2011, in prep.) we developed a different approach: We introduce a radio power probability distribution which give the probability to find a relic with a given luminosity for a cluster with given mass and redshift. Motivated by simulations we adopt a log-normal distribution, with scaling laws depending on cluster mass and redshift.

However, several rather bright radio relics have been discovered only recently, hence, the flux threshold above which the current list of relics is complete would be at least a few hundred mJy, leaving only a few relics in the flux-complete list. Instead of using a sharp flux threshold we introduced the discovery probability, which gives a smooth transition between the non-discovery and the discovery of relics. The parameters of the discovery probability can only be estimated since we do not know how many rather bright relics we are not yet detected. In Nuza et al. (2011, in prep.) we present some plausible estimates.

It is impossible to predict the number of observable relics purely based on simulations, mainly because we can only speculate about the electron acceleration efficiency. We therefore normalize the predicted number counts with the number of observed relics, see Fig. 4. As a result, we can infer expected number counts for future low frequency surveys. In Nuza et al. (2011, in prep.) we show that both, the LOFAR-120 MHz-Tier 1 survey and the proposed WODAN survey with the upcoming APERTIF sensors at WSRT, have the potential to detect of the order of 1000 radio relics. Crucial for actually confirming the relics candidates is that the corresponding galaxy clusters need to be identified, since most of the low luminosity relics reside in clusters with low X-ray brightness. More precisely, we predict that $50 \%$ of the relics with a flux above $1 \mathrm{mJy}$ at $1.4 \mathrm{GHz}$ reside in clusters with an X-ray flux below $10^{-12} \mathrm{erg} \mathrm{s}^{-1} \mathrm{~cm}^{-2}$.

In a recent work van Weeren et al. (2011, submitted) selected 544 clusters from the NORAS and the REFLEX cluster samples with an X-ray flux above $3 \times 10^{-12} \mathrm{erg} \mathrm{s}^{-1} \mathrm{~cm}^{-2}$ which are located outside the galactic plane. For 17 of the clusters a radio relic has been reported in the literature. Based on this cluster sample van Weeren et al. (2011) find tentative evidence that the fraction of clusters which host a relic increases with both, cluster X-ray luminosity and redshift. The large relic samples expected from the upcoming low frequency surveys will allow tight constraints on such behaviour.

\section{Summary}

Relics have been found in about three dozen clusters. They are believed to originate from merger shocks in clusters. One plausible scenario for relic formation is that a small fraction of thermal electrons gets accelerated at the shock front to relativistic energies. Bound to the gas flow by small

gyroradii the relativistic electrons advect downstream and emit synchrotron 


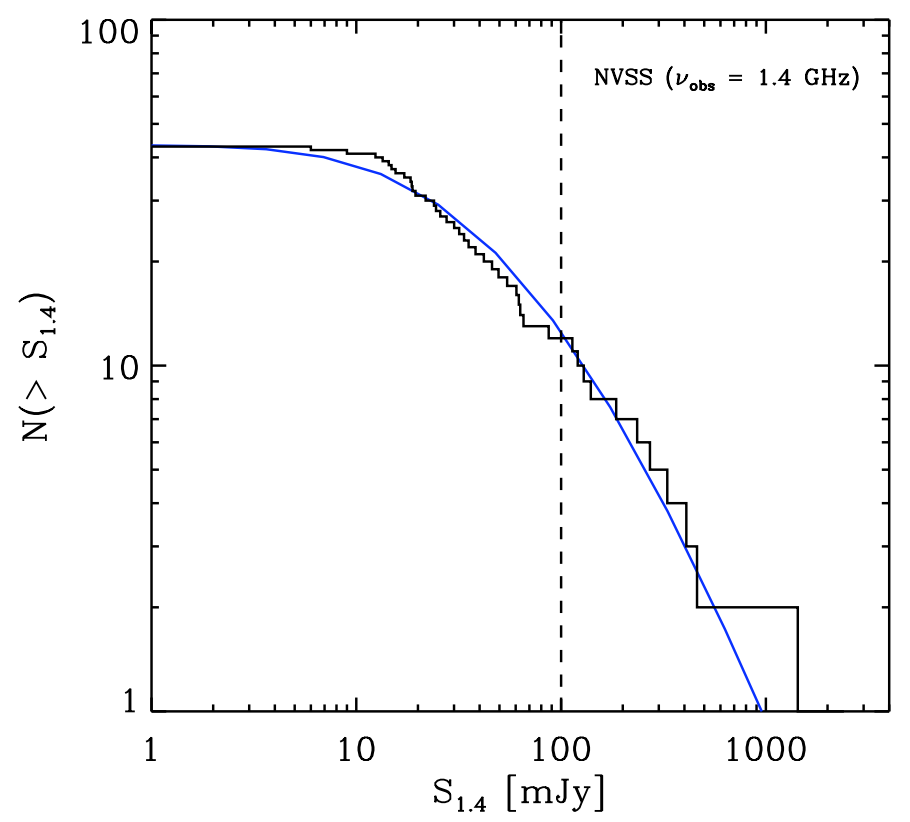

Figure 4. Number counts $N\left(>S_{1.4}\right)$, where $S_{1.4}$ is the radio flux of one or more relics within one cluster. In Nuza et al. (2011, in prep.) we compile a list of all clusters for which one or more relics are known. The stepped curve shows the cumulative number counts, while the solid line shows the fit obtained from radio power probability distribution, after normalizing to the total number of known relics.

radiation. Hydrodynamical cosmological simulations allow to study merger shocks in detail. To study as well the formation of radio relics a sub-grid model for both, the electron acceleration and the downstream cooling is needed. The relic in CIZA 2242 shows that the intrinsic width is of the order of 10 to $50 \mathrm{kpc}$. Hence, current cosmological simulations are close to spatially resolving the downstream cooling, but in our case a sub-grid module for it is justified.

We presented a high resolution simulation of a cluster merger. The resulting shock front shows a complex surface: There are many 'wiggles' on the surface which are caused by the inhomogeneity of the ICM. Temperature and density variations lead to a not uniform shock speed. The upstream temperature affects the Mach number. We determined the Mach number distribution of the shock front and find that it peaks at the value expected from the X-ray jump, namely 2.5. Moreover, we find a rather broad distribution of Mach numbers, up to 4.5. Since the actual radio luminosity is believed to vary strongly for Mach numbers from 2 to 5 the luminosity is very inhomogeneously distributed across the shock surface.

It would be nice to develop a 'radio luminosity function of shock fronts'. However, the high resolution merger simulation illustrates that the shock 
fronts can be patchy, with low surface brightness regions between more luminous ones. As a consequence, in Nuza et al. (2011, in prep.) we introduce the radio power probability distribution, which relates the radio power of relics in a cluster to the cluster mass instead of the shock surface. Since the actual electron acceleration efficiency is not known we normalize the probability distribution by the number of observed relic. As a result we find the LOFAR-120 MHz-Tier 1 survey could detect more than a thousand relics.

ACKNOWledgements. SEN and MH thank Gustavo Yepes for providing access to the Mare Nostrum Universe. Simulations were carried out at the BSC-CNS (Barcelona, Spain) and at the Leibniz-Rechenzentrum (Munich, Germany). MH and MB acknowledge support by the research group FOR 1254 'Magnetisation of Interstellar and Intergalactic Media: The Prospects of Low-Frequency Radio Observations' founded by the Deutsche Forschungsgemeinschaft.

\section{References}

Bagchi, J., Durret, F., Neto, G. B. L., \& Paul, S. 2006, Science, 314, 791

Bagchi, J., Sirothia, S. K., Werner, N., Pandge, M. B., Kantharia, N. G., H, IshwarChandra C., Gopal-Krishna, Paul, S., \& Joshi, S., 2011, ApJ 736, L8

Blandford, R. \& Eichler, D. 1987, Phys. Rep., 154, 1

Bonafede, A., Feretti, L., Murgia, M., Govoni, F., Giovannini, G., Dallacasa, D., Dolag, K., \& Taylor, G. B. 2010, A \&6 A, 513, 30

Drury, L. O. 1983, Reports of Progress in Physics, 46, 973

Enßlin, T. A., Biermann, P. L., Klein, U., \& Kohle, S. 1998, A \& A, 332, 395

Hoeft, M., \& Brüggen, M. 2007, MNRAS, 375, 77

Hoeft, M., Brüggen, M., Yepes, G., Gottlöber, S., \& Schwope, A. 2008, MNRAS, 391, 1511

Keshet, U. 2010, arXiv, 1011.0729

Malkov, M. A. \& OC Drury, L. 2001, Reports on Progress in Physics, 64, 429

Markevitch, M. 2010, arXiv 1010.3660

Nakazawa, K., Sarazin, C. L., Kawaharada, M., et al. 2009, PASJ, 61, 339

Nuza, S. E., Hoeft, M., van Weeren, R. J., Gottloeber, S., Yepes, G., 2011, arXiv 1111.1721

Paul S., Iapichino L., Miniati F., Bagchi J., Mannheim K., 2011, ApJ, 726, 17

Pfrommer C., Springel V., Enlin T. A., Jubelgas M., 2006, MNRAS, 367, 113

Röttgering, H. J. A., Wieringa, M. H., Hunstead, R. W., \& Ekers, R. D. 1997, MNRAS, 290, 577

Ryu D., Kang H., Hallman E., Jones T. W., 2003, ApJ, 593, 599

Springel, V. 2005, MNRAS, 364, 1105

Treumann, R. A. 2009, A\&ARv, 17, 409

Vazza, F., Brunetti, G., Gheller, C. 2009, MNRAS, 395, 1333

van Weeren, R. J., Röttgering, H. J. A., Brüggen, M., \& Hoeft, M. 2010, Science, 330, 347 